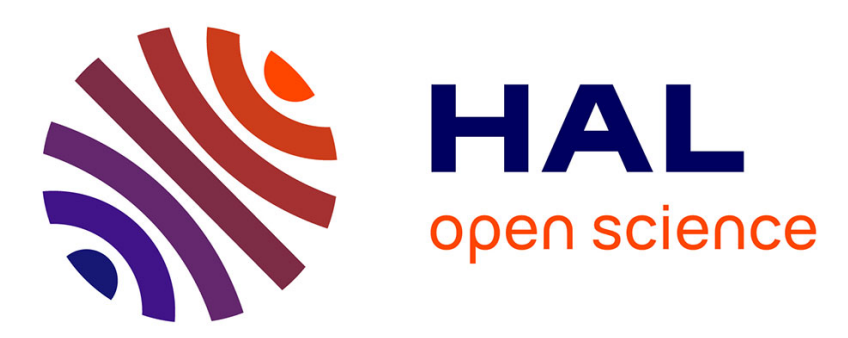

\title{
Fourier optics heuristics for diffraction at infinity by an index discontinuity in a one-dimensional slab
}

\author{
Marius Peloux, Jean-Paul Hugonin, Pierre Chavel
}

\section{To cite this version:}

Marius Peloux, Jean-Paul Hugonin, Pierre Chavel. Fourier optics heuristics for diffraction at infinity by an index discontinuity in a one-dimensional slab. Journal of the Optical Society of America. A Optics, Image Science, and Vision, 2011, 28 (8), pp.1648-1655. hal-00860981

\section{HAL Id: hal-00860981 \\ https://hal-iogs.archives-ouvertes.fr/hal-00860981}

Submitted on 11 Sep 2013

HAL is a multi-disciplinary open access archive for the deposit and dissemination of scientific research documents, whether they are published or not. The documents may come from teaching and research institutions in France or abroad, or from public or private research centers.
L'archive ouverte pluridisciplinaire HAL, est destinée au dépôt et à la diffusion de documents scientifiques de niveau recherche, publiés ou non, émanant des établissements d'enseignement et de recherche français ou étrangers, des laboratoires publics ou privés. 


\title{
Fourier optics heuristics for diffraction at infinity by an index discontinuity in a one-dimensional slab
}

\author{
Marius Peloux, ${ }^{1,2, *}$ Jean-Paul Hugonin, ${ }^{1}$ and Pierre Chavel $^{1}$ \\ ${ }^{1}$ Laboratoire Charles Fabry de l'Institut d'Optique, Université Paris-Sud, CNRS, \\ Campus Polytechnique, RD 128, 91127 Palaiseau, France \\ ${ }^{2}$ Essilor International, 81 Boulevard Jean-Baptiste Oudry, 94000, Créteil, France \\ ${ }^{*}$ Corresponding author: marius.peloux@institutoptique.fr
}

Received May 25, 2011; accepted June 17, 2011;

posted June 21, 2011 (Doc. ID 148219); published July 15, 2011

\begin{abstract}
We study the far-field reflected diffraction pattern of an index discontinuity in a thin one-dimensional slab illuminated by a plane wave and show that a time-saving modeling technique based on plane wave expansion approaches fairly well the Maxwell-based rigorous models. This method is simple to implement, and it furthermore allows a good understanding of the optical phenomena involved in the propagation of light through the slab. (C) 2011 Optical Society of America
\end{abstract}

OCIS codes: $\quad 050.1940,050.1970,070.7345,080.1510,230.3990$.

\section{INTRODUCTION}

This work was motivated by the desire to account for parasitic optical effects in pixelated optical components. As technologies to fabricate pixels on various kinds of substrates develop, pixelated components present themselves as approximants to continuous display or wavefront shaping components. The visibility of the discontinuities between pixels is a parasitic effect that should be minimized [1-3]. Specifically, we would like to quantitatively account for the parasitic shine effects that appear when one observes a pixelated component from a distance and that result from diffraction at the pixel boundaries. Indeed, pixelated components show abrupt edges perpendicular to the substrate, which may typically be either a transition from the index substrate (after etching) to air or a transition between one medium deposited on the substrate to another such medium with a different index. As a generic building block of the discontinuity between pixels, we therefore selected the index discontinuity in a one-dimensional (1D) slab. The purpose of this work is to introduce a heuristic model for diffraction by an index discontinuity in a $1 \mathrm{D}$ slab that provides results close to the true electromagnetic phenomena in a number of cases of practical interest but can be calculated in a short time so that complex components comprising many pixels can be modeled.

Indeed, in spite of considerable progress in the numerical solution of the Maxwell's equations, and in spite of their broad applicability to many component geometries from gratings to nanophotonic devices, approximate solutions are still useful because they offer faster numerical implementation. Among them, heuristic solutions, which can be described in terms of simple and well-understood optical phenomena such as the optical path length along a ray or Fresnel reflection and transmission, have the additional advantage that they lend themselves easily to intuition and are therefore good guides to component design.

The heuristic approach was pioneered many years ago, notably by Swanson [4], who introduced the so-called extended scalar theory to account for the diffraction efficiency of periodic structures such as echelette or multilevel binary gratings. It can be seen as an intermediate approach between the crude "scalar theory" and the elaborate but computationally extensive rigorous approach, which explicitly solves the Maxwell's equations.

The so-called "scalar" theory, also called "thin element approximation," assumes the phase delay through a layer of thickness $H$ of an index $n$ medium to be $2 \pi(n-1) H / \lambda$ for wavelength $\lambda$ irrespective of the incidence and neglects the shadowing that occurs when light reaches boundaries between grooves of a grating or pixels of a pixelated element. Those two fairly crude approximations provide acceptable results when characteristic lengths on the component are much larger than a wavelength, such as, for example, a ruled grating with a pitch of tens or hundreds of wavelengths. For that reason, the diffraction angles involved are small; therefore, the scalar approximation is valid mainly in the paraxial domain. Rigorous methods are, in principle, applicable at all length scales but become impractical because of the computational burden as soon as the total dimension of the component reaches just a few wavelengths. Investigating the diffraction by echelette and multilevel binary gratings [4], Swanson took into account the real geometric path length across the structure as well as the shadowing effect that occurs when the geometric ray hits the echelette profile discontinuity perpendicular to the substrate. While that refinement brings little for gratings with a pitch much larger than the wavelength and fails for a pitch close to or smaller than the wavelength, it brings a clear progress in the intermediate case of a pitch somewhat larger than the wavelength. Glytsis [5] further analyzed the limits of the scalar theory to periodic diffractive optical elements. References [6-9] detail calculation techniques that imply both geometric and Fourier optics and lead to acceptable results in the nonparaxial domain, and the inclusion of knife-edge diffraction has proven useful for near-field modeling of elements a few wavelengths thick [10]. In Ref. [11], the 
diffraction pattern of a whole grating is obtained by the coherent summation of the field diffracted by all periods of the grating, each being calculated beforehand using rigorous Maxwell-based models.

The discontinuity in the $1 \mathrm{D}$ slab that we consider here obviously cannot be analyzed by the scalar theory, which just ignores the boundary effects that we are interested in. Grating models are also not directly applicable, because the structure is inherently nonperiodic. We shall show that a heuristic approach nevertheless exists for our case. Namely, we introduce the plane wave expansion [12], also called the "angular spectrum representation," as an additional tool in the domain of heuristic models. Fresnel transmission and reflection coefficients have to be taken into account as well. However, because we consider the spurious effects of pixel edges observed from centimeters to meters away, near-field effects, in particular those carried by evanescent components of the field, are irrelevant to our discussion and will not be considered here. On the contrary, diffraction at infinity will provide a fair estimation of the effects. It is therefore our goal to show that in the case of diffraction at infinity by an index discontinuity in a $1 \mathrm{D}$ slab illuminated by a plane wave, a heuristic model that relies only on Fourier optics can be a good approximation to "rigorous" results whenever (just like in Swanson's work) no length scale close to or smaller than one wavelength is involved.

We assess the validity of our model by testing it against electromagnetic calculations. For the latter, we use our latest implementation of the well-known rigorous coupled wave analysis (RCWA) [13] in its generalization to nonperiodic structures, the aperiodic Fourier modal method (a-FMM) [14]. One may note here that the comparison is possible because we consider just one index discontinuity in a $1 \mathrm{D}$ slab. Our heuristic model can be extended to components comprising many pixels and therefore many discontinuities, while the electromagnetic methods would lead to impractically heavy calculations.

In Section 2, we first describe the diffracting structure that we study in this work and identify the quantity of interest to address diffraction at infinity. We also summarize in a few lines the two electromagnetic implementations of the a-FMM that we have used here as references. Our heuristic model is detailed in Section 3 . Section $\underline{4}$ shows a selection of numerical results, and the limitations are discussed in Section $\underline{5}$. This manuscript is restricted to TE polarization and to phenomena observed in reflection. As we have found out through additional tests that are not reported here for the sake of brevity, no obstacle exists for its extension to TM polarization and to transmission.

\section{TWO APPROACHES FOR A SLAB DIFFRACTION PROBLEM}

The structure considered is shown in Figs. $\underline{1}$ and $\underline{2}$, where $O$ is the center of the orthogonal coordinate system $(O, x, z)$. A monochromatic plane wave propagates in an incident medium of index $n_{0}=1$ and then hits an interface with a structure whose dimension along the $z$ axis is $H$ and which is infinite along the $x$ axis. This structure is deposited upon a substrate of index $n_{3}$, which is semi-infinite in the $z<-H$ half-space, and it is divided into two parts for $-H<z<0$ : the refraction index is $n_{1}$ for $x<0$ and $n_{2}$ for $x>0$. A two-dimensional geo-
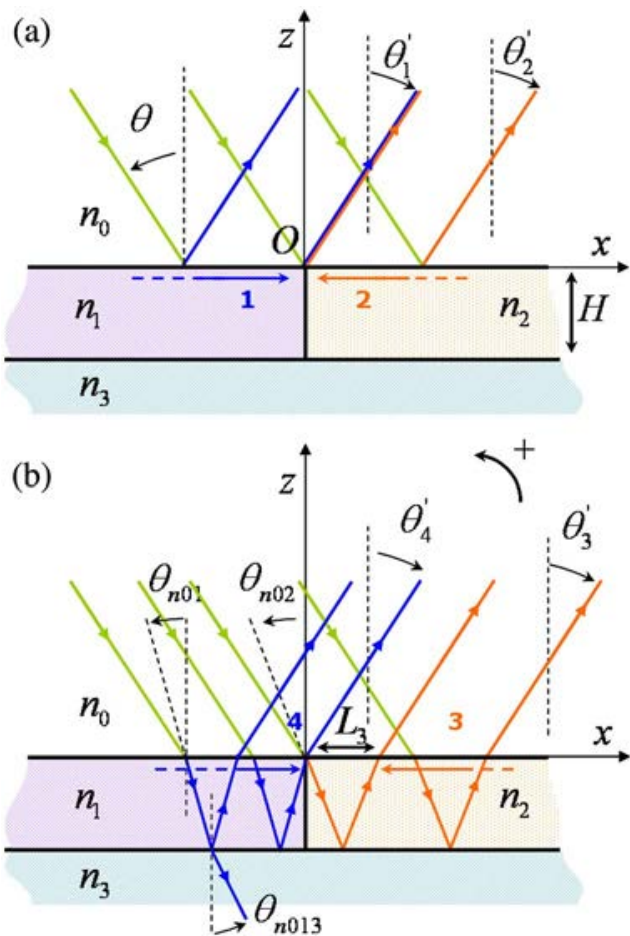

Fig. 1. (Color online) (a) Partial waves $1(x<0)$ and $2(x>0)$ and (b) partial waves $3\left[x>2 H \tan \left(\theta_{n 02}\right)\right]$ and $4(x<0)$. Partial waves 1 and 4 have semi-infinite pupils on the left, and partial waves 2 and 3 have semi-infinite pupils on the right.

metry is considered; i.e., the structure is invariant under any translation in the $y$ direction, and the incoming light is a plane wave with its wave vector $\vec{k}$ in the $x, z$ plane. Its amplitude
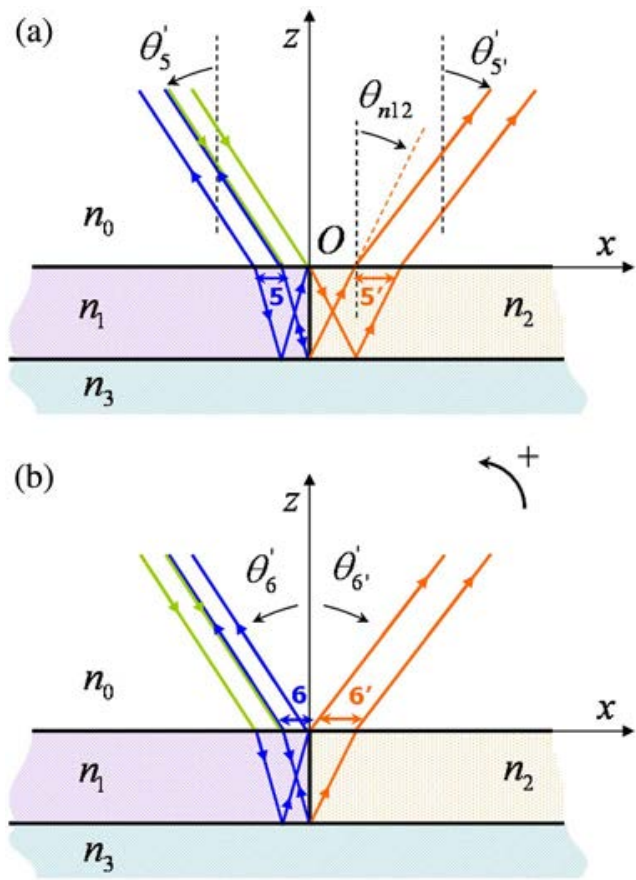

Fig. 2. (Color online) (a) Partial waves $5\left[-2 H \tan \left(\theta_{n 01}\right)<\right.$ $\left.x<-H \tan \left(\theta_{n 01}\right)\right]$ and $5^{\prime}\left[H \tan \left(\theta_{n 12}\right)<x<2 H \tan \left(\theta_{n 12}\right)\right]$ and (b) partial waves $6\left[-H \tan \left(\theta_{n 01}\right)<x<0\right]$ and $6^{\prime}\left[0<x<H \tan \left(\theta_{n 12}\right)\right]$. Partial waves $5,6,5^{\prime}$, and $6^{\prime}$ are limited by the vertical index discontinuity in the slab and therefore have finite-size pupils. 
is denoted $u_{0}$, and $\theta$ is its incidence angle. For numerical applications, we consider a wavelength $\lambda=500 \mathrm{~nm}$.

As explained in the introduction, we are interested in diffraction at infinity by that structure, and we seek to give a good quantitative account of the effect of such a structure for various values of parameters $n_{0}, n_{1}, n_{2}, n_{3}$, and $H / \lambda$. To that end, we compare an electromagnetic approach taken as the reference and the heuristic approach, which is the subject of the present work.

In both cases, we start from an expression of the angular spectrum of the diffracted field $u\left(x, 0_{+}\right)$calculated just above the structure $\left(z=0_{+}\right.$, index $\left.n_{0}\right)$, where the + index designates the limit as value $z=0$ is approached from the positive side. The angular spectrum is the Fourier transform $\tilde{u}(\alpha / \lambda)$ of $u\left(x, 0_{+}\right)$. We discard its evanescent part and express the diffraction at angle $\theta^{\prime}=\arcsin (\alpha)$ by $u_{\infty}(\alpha)$. The angular spectrum method relates $u_{\infty}(\alpha)$ to $\tilde{u}(\alpha / \lambda)$ through [15]

$$
u_{\infty}(\alpha) \propto \sqrt{\frac{1-\alpha^{2}}{\lambda} \tilde{u}}\left(\frac{\alpha}{\lambda}\right) .
$$

Equation (1) is valid for both the electromagnetic approach and our heuristic method. Therefore, each method is aimed at calculating the complex amplitude repartition $u\left(x, 0_{+}\right)$or at least its nonevanescent part, before using Eq. (1) to evaluate the far-field light distribution. In the next few lines, we briefly describe the electromagnetic algorithms that we implemented before describing our heuristic method in Section 3 .

In the electromagnetic approach, the field is obtained by rigorously solving the Maxwell's equations using the method described in [14], relying on an analytical integration along the $z$ or $x$ direction and on the use of a nonlinear complex coordinate transform which maps the two semi-infinite intervals to finite intervals. Because of the coordinate transform, the fields are nil at the boundaries of the computational box and can be periodized and expanded in a Fourier series: that is "artificial periodization."

The method used to generate the incident plane wave depends on the direction of integration. We may select either $z$ or $x$ as the direction of integration, which splits the electromagnetic method into two mathematically equivalent but numerically independent subcases. In the $z$-integration method, we introduce a "discontinuity plane" $z=z_{0}$ in the upper region. In the $x$-integration method, we introduce a line of pseudoperiodical point sources. The complex amplitudes of the discontinuity and those of the sources are chosen so that the generated incident wave is "almost" plane at the origin and decreases slowly at infinity, in order to avoid numerical truncation artifacts. The relatively large (a few wavelengths) size of the objects studied here makes the convergence of these rigorous methods delicate, but the fact that our two integration methods lead to perfectly overlapped curves confirms the results. The detail of these two implementations of the a-FMM will be the object of a future publication.

When $x \rightarrow \pm \infty$, the field $u$ has an asymptotic form given by geometric optics and multiple beams interferences, as it is a simple reflection of the incident plane wave on a slab. To avoid numerical artifacts due to truncation, we subtract this asymptotic expression from the field $u$ before the Fourier integration, and we add its Fourier transform (in the sense of distributions) back to the final result.

\section{DESCRIPTION OF THE MODEL}

In the heuristic method that we develop here that we call the Fourier optics model (FOM), we consider eight partial waves denoted $1,2,3,4,5,5^{\prime}, 6$, and $6^{\prime}$, whose size and position are suggested by the rays in Figs. $\underline{1}$ and $\underline{2}$. Note that on these figures, the partial waves are represented as geometric optic rays for the sake of convenient visualization but that the calculations involve wave optics rather than just geometric optics. Each "case" 1, 2, 3, 4, 5, 5', 6, 6' corresponds to different Fresnel reflection or transmission coefficients, phase delays or wave vectors $\vec{k}$. The primed numbers refer to waves transmitted at the slab end interface between $n_{1}$ and $n_{2}$ at $x=0$, while the others are either reflected at that interface (5 and 6), or not affected by it (1, 2, 3, and 4).

In this paper we will use the notation $n_{p}-n_{q}$ to designate an interface with light coming from the $n_{p}$ side. We introduce the following notations for the angles involved in the calculations, the sign convention being shown in Figs. $\underline{1}$ and $\underline{2}$, where we draw a geometric representation of light propagation in the slab. All angles are measured from the normal to the substrate. If we consider an incidence angle $\theta, \theta_{n 01}$ (respectively, $\theta_{n 02}$ ) is the angle in the $n_{1}$ index medium (respectively, $n_{2}$ ) after refraction at the $n_{0}-n_{1}$ interface (respectively, $n_{0}-n_{2}$ ). After refraction at $n_{1}-n_{2}$, the refraction angle in $n_{2}$ is $\theta_{n 12}$, and, in the absence of total internal reflection, we denote by $\theta_{n 013}$ the refraction angle in $n_{3}$ after refraction at $n_{1}-n_{3}$. The angle for geometric rays reflected to infinity for the case $j$ is denoted $\theta_{j}^{\prime}$. It is clear that many of the $\theta_{j}^{\prime}$ are equal to each other, but we keep the notations separate to identify which partial wave is being considered. We expect to see in our results some diffraction peaks located around the different values calculated for $\theta_{j}^{\prime}$.

For simplicity's sake, we restrict our calculations to positive values of $\theta$. Indeed, changing the sign of $\theta$ is equivalent to interchanging the values of $n_{1}$ and $n_{2}$. All these angles are related to each other via the Snell-Descartes laws, with the peculiarity that, remembering that we refer all angles to the normal to the substrate, $n_{1} \cos \left(\theta_{n 01}\right)=n_{2} \cos \left(\theta_{n 12}\right)$. We assume that $n_{0}=1, n_{1} \geq 1$, and $n_{2} \geq 1$ such that there is no total reflection at the first interface met by the incident wave. In fact, the case where total reflection may occur could be treated along exactly the same lines. We also neglect all beams that are reflected more than once at the interface with the substrate, which is why the problem is reduced to those eight partial waves only.

We investigate, in detail, the study of the propagation of partial wave 3, and we give in Appendix A the results following the same method for the seven other cases. Let us consider in Fig. 3 an unfolded scheme associated to the propagation of partial wave 3, on which the light is coming from the back side of the slab $(z=-2 H)$ and which is equivalent to the one depicted in Fig. 1(b), taking into account the reflection coefficient at $z=-H$, which corresponds to the $n_{2}-n_{3}$ interface. We start from the complex amplitude repartition just above the $z=-2 H$ interface, which we denote as $z=-2 H_{+}: u(x$, $\left.-2 H_{+}\right)$:

$$
u_{3}\left(x,-2 H_{+}\right)=\operatorname{He}(x) e^{i \frac{2 \pi}{\lambda} n_{0} x \sin \theta} t_{02}(\theta),
$$

where $\operatorname{He}(x)$ is the Heaviside function that describes the semi-infinite pupil of partial wave 3 and $t_{02}(\theta)$ is the Fresnel transmission coefficient at $n_{0}-n_{2}$ under incidence $\theta$ : 


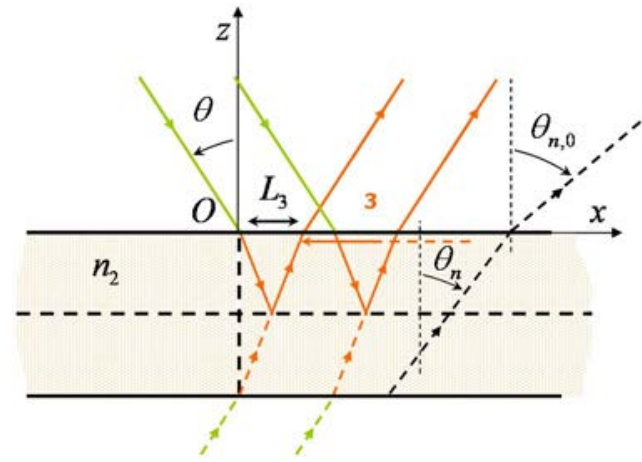

Fig. 3. (Color online) Unfolded scheme associated to the study of partial wave 3 . The thick vertical dashed lines stand for the $n_{1}-n_{2}$ discontinuity, and the horizontal dashed lines stand for the interface between the slab and the substrate.

$$
t_{02}^{\mathrm{TE}}(\theta)=\frac{2 n_{0} \cos (\theta)}{n_{0} \cos (\theta)+n_{2} \cos \left(\theta_{n 02}\right)}
$$

The Fourier transform of $u_{3}\left(x,-2 H_{+}\right)$at spatial frequency $\mu$ is given by

$$
\tilde{u}_{3,-2 H}(\mu)=\frac{t_{02}(\theta)}{2 \pi i\left(\mu-n_{0} \sin \theta / \lambda\right)} .
$$

Let us give an expression for the complex amplitude repartition $u_{3, r}$ in the $n_{2}$ index medium defined by $-2 H<z<-H$. The plane wave decomposition leads to

$$
u_{3, r}(x, z)=\int \tilde{u}_{3,-2 H}\left(\frac{\alpha_{n}^{\prime}}{\lambda}\right) e^{i \frac{2 \pi}{\lambda}(2 H+z) \sqrt{n_{2}^{2}-\alpha_{n}^{\prime 2}}} e^{i \frac{2 \pi}{\lambda} x \alpha_{n}^{\prime}} \frac{\mathrm{d} \alpha_{n}^{\prime}}{\lambda},
$$

$\alpha_{n}^{\prime}=n_{2} \sin \theta_{n}$, where the angles $\theta_{n}$, one of which is represented in Fig. 3, are associated to the propagation direction of the plane waves. The terms in the exponential correspond to $i \vec{k}_{n_{2}} \cdot \vec{r}$, where $\vec{r}$ is the position vector whose coordinates are $(x, z)$ in the $(O, x, z)$ coordinate system and $\vec{k}_{n_{2}}$ is the wave vector of a plane wave in the $n_{2}$ index medium. $u_{3, r}$ becomes $u_{3, R}$ in $H<z<0$ after reflection at $n_{2}-n_{3}$ :

$$
u_{3, R}(x, \boldsymbol{z})=\int r_{23}\left(\alpha_{n}^{\prime}\right) \tilde{u}_{3,-2 H}\left(\frac{\alpha_{n}^{\prime}}{\lambda}\right) e^{i \frac{2 \pi}{\lambda}(2 H+z) \sqrt{n_{2}^{2}-\alpha_{n}^{\prime 2}}} e^{i \frac{2 \pi}{\lambda} x \alpha_{n}^{\prime}} \frac{d \alpha_{n}^{\prime}}{\lambda},
$$

where $r_{23}\left(\alpha_{n}^{\prime}\right)$ is the Fresnel coefficient for the reflection of the plane wave associated to $\alpha_{n}^{\prime}$. When this plane wave meets the $n_{2}-n_{3}$ interface, its transmitted part is refracted at an angle of $\theta_{n, 3}$, not represented in Fig. 3 , such that according to the Snell-Descartes' law for refraction: $n_{2} \sin \theta_{n}=n_{3} \sin \theta_{n, 3}$. Then

$$
r_{23, \mathrm{TE}}\left(\alpha_{n}^{\prime}\right)=\frac{n_{2} \cos \theta_{n}-n_{3} \cos \theta_{n, 3}}{n_{2} \cos \theta_{n}+n_{3} \cos \theta_{n, 3}}=\frac{\sqrt{n_{2}^{2}-\alpha_{n}^{\prime 2}}-\sqrt{n_{3}^{2}-\alpha_{n}^{\prime 2}}}{\sqrt{n_{2}^{2}-\alpha_{n}^{\prime 2}}+\sqrt{n_{3}^{2}-\alpha_{n}^{\prime 2}}} .
$$

We can notice that if $n_{3}<n_{2}, r_{23, \mathrm{TE}}\left(\alpha_{n}^{\prime}\right)$ has complex values for the higher $\alpha_{n}^{\prime}$ : that is total internal reflection. After refraction at $n_{2}-n_{0}, u_{3, R}$ becomes $u_{3, R T}$ in $z>0$. According to the Snell-Descartes' law, the component of the wave vector changes along $z$ so that its norm is equal to $2 \pi n_{0} / \lambda$ in the $n_{0}$ half-space:

$$
\begin{aligned}
u_{3, R T}(x, z)= & \int t_{20}\left(\alpha_{n}^{\prime}\right) r_{23}\left(\alpha_{n}^{\prime}\right) \tilde{u}_{3,-2 H}\left(\frac{\alpha_{n}^{\prime}}{\lambda}\right) \\
& \times e^{i \frac{2 \pi}{\lambda} 2 H \sqrt{n_{2}^{2}-\alpha_{n}^{\prime 2}}} e^{i \frac{2 \pi}{\lambda} z \sqrt{n_{0}^{2}-\alpha_{n}^{\prime 2}}} e^{i \frac{2 \pi}{\lambda} x \alpha_{n}^{\prime}} \frac{\mathrm{d} \alpha_{n}^{\prime}}{\lambda},
\end{aligned}
$$

where in this medium $\alpha_{n}^{\prime}=n_{0} \sin \theta_{n, 0}=n_{2} \sin \theta_{n}$. The highest values of $\theta_{n}$ such that $n_{2} \sin \theta_{n}>1$ are associated to total internal reflection and are thus not taken into account in Eq. (8). Therefore, $-1 \leq \alpha_{n}^{\prime} \leq 1$, and

$$
t_{20, \mathrm{TE}}\left(\alpha_{n}^{\prime}\right)=\frac{2 n_{2} \cos \theta_{n}}{n_{2} \cos \theta_{n}+n_{0} \cos \theta_{n, 0}}=\frac{2 \sqrt{n_{2}^{2}-\alpha_{n}^{\prime 2}}}{\sqrt{n_{2}^{2}-\alpha_{n}^{\prime 2}}+\sqrt{n_{0}^{2}-\alpha_{n}^{\prime 2}}} .
$$

Here the expression for $u\left(x, 0_{+}\right)$in Eq. (1) is $u_{3, R T}\left(x, 0_{+}\right)$, whose analytical expression given by Eq. (8) reveals an inverse Fourier transform. Thus, one can write the repartition at infinity $u_{3, \infty}(\alpha)$ :

$$
u_{3, \infty}(\alpha) \propto \sqrt{\frac{1-\alpha^{2}}{\lambda}} t_{20}(\alpha) r_{23}(\alpha) \tilde{u}_{3,-2 H}\left(\frac{\alpha}{\lambda}\right) e^{i \frac{2 \pi}{\lambda} 2 H \sqrt{n_{2}^{2}-\alpha^{2}}}
$$

The exponential term corresponds to a phase delay due to the double crossing of the $n_{2}$ medium. In Appendix A, we show that the calculations associated to the other partial waves are expressed in the same vein. This means that the repartition of the complex amplitude at infinity can be calculated using a single Fourier transform for each partial wave, which only involves analytical expressions of phase delays and Fresnel coefficients. As a result, the calculation time is reduced by orders of magnitude compared to the electromagnetic models. Moreover, with such an approach, we can easily calculate the contribution of each partial wave to the total diffracted energy, which cannot be done using the a-FMM model and leads to a better understanding of the diffraction phenomenon in the slab.

We underline the fact that when the angle refracted at a given interface using the Snell-Descartes' law has an imaginary value, it indicates total internal reflection. The associated Fresnel coefficient in reflection is then also an imaginary number, whose argument corresponds to a phase shift, that we calculate using imaginary cosines, and we include those contributions in our model. Therefore, our model is applicable to the cases where total internal reflection is involved. Of course, for the transmitted part of such partial waves, we could take into account the evanescent waves, but because we are interested in the far-field diffraction pattern, we omit them. This restriction is illustrated by the fact that we restrict the range of values of $\alpha_{n}^{\prime}$ from $-n_{2} \leq \alpha_{n}^{\prime} \leq n_{2}$ to $-1 \leq \alpha_{n}^{\prime} \leq 1$ between Eqs. (ㅁ) and (ㅁ). The next section illustrates the validity of our approach.

\section{RESULTS}

As the light considered here is monochromatic, in Figs. $\underline{4}-\underline{6}$ we represent $\left|\sqrt{1-\alpha^{2}} u_{\infty}(\alpha)\right|^{2}$, expressed in square micrometers. There, as examples among a number of cases that we have 


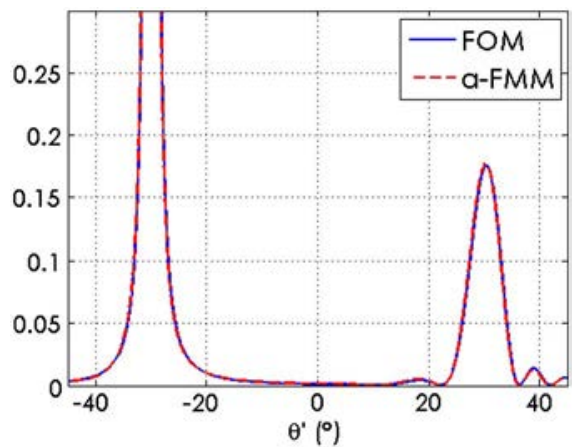

Fig. 4. (Color online) TE mode $\left(n_{1}=1, n_{2}=1.5, n_{3}=1.5, H=4 \mu \mathrm{m}\right.$, $\theta=30^{\circ}$ ). Central peak associated to cases 1 to 4 and peak associated to cases 5 and 6 . The specular peak grows to infinity at $\theta^{\prime}=-30^{\circ}$, as should be expected, and it has therefore been truncated.

investigated, we show the reflected angular diffraction patterns corresponding to some specific angular regions of diffraction at infinity for two sets of parameters $\left(n_{1}, n_{2}, n_{3}\right.$, $H, \theta)$ in the TE mode, calculated with our FOM and with the a-FMM methods. Because the problem is unbounded in $x$ on the negative and the positive sides, the central reflection peak obviously grows to infinity and has therefore been cut out. We can verify that the peaks appear where they were expected to. Indeed, for instance, in the case of Fig. 5, for $\theta=10^{\circ}$ the specular peak associated to cases $1,2,3$, and 4 is located around $-10^{\circ}$, and the peak associated to cases 5 and 6 around $10^{\circ}$. We can also calculate that $\theta_{5^{\prime}}^{\prime}=$ $\theta_{6^{\prime}}^{\prime} \simeq-34.4^{\circ}$ and check that this value agrees with the calculated diffraction pattern. In Fig. $\underline{6}$ we illustrate a configuration where no light is transmitted through the $n_{1}-n_{2}$ interface as the angle $\theta_{n 12}$ has an imaginary value. The resemblance between the curves obtained with our FOM and by the aFMM methods and the absence of a peak associated to partial waves $5^{\prime}$ and $6^{\prime}$ proves that our model is valid even in the presence of total internal reflection.

In this latter configuration where no light associated to partial waves $5^{\prime}$ and $6^{\prime}$ is reflected toward infinity, we also show in Fig. 7 a magnification around the peak associated to partial waves $\overline{5}$ and 6 , and we represent the results calculated considering, respectively, partial wave 5 only, partial wave 6 only, partial waves 1 to 4 only, and all six partial waves $1,2,3,4,5$, and 6 . We can see that partial waves 1 to 4 have a weak in-

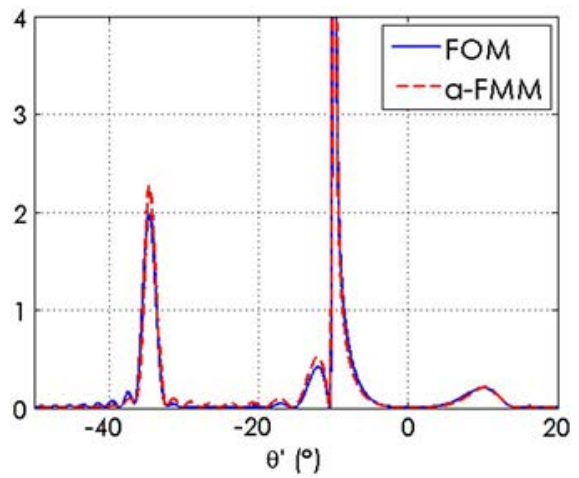

Fig. 5. (Color online) TE mode $\left(n_{1}=1.4, n_{2}=1.5, \quad n_{3}=1\right.$, $\left.H=20 \mu \mathrm{m}, \theta=10^{\circ}\right)$. Left (near $-34.4^{\circ}$ ): peak associated to cases 5 and $6^{\prime}$, middle (near $-10^{\circ}$ ): central peak associated to cases 1 to 4 and right (near $10^{\circ}$ ): peak associated to cases 5 and 6 . The central peak grows to infinity at $\theta^{\prime}=-10^{\circ}$, as should be expected, and it has therefore been truncated.

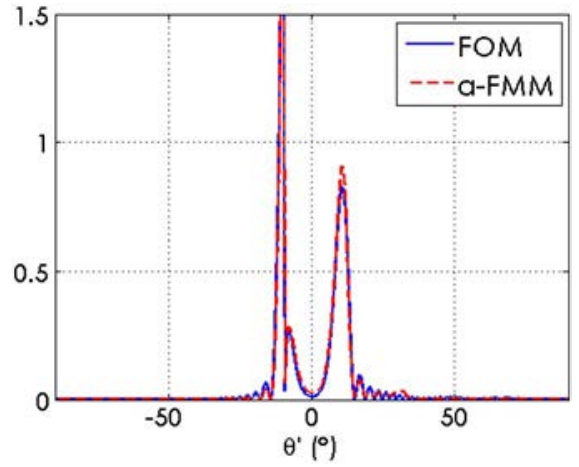

Fig. 6. (Color online) TE mode $\left(n_{1}=1.5, n_{2}=1.4, \quad n_{3}=1\right.$, $H=20 \mu \mathrm{m}, \theta=10^{\circ}$ ). Left (near $-10^{\circ}$ ): central peak associated to cases 1 to 4 and right (near $10^{\circ}$ ): peak associated to cases 5 and 6 . The central peak grows to infinity at $\theta^{\prime}=-10^{\circ}$, as should be expected, and it has therefore been truncated.

fluence on the shape of the peak around $\theta^{\prime}=10^{\circ}$, because their intensity in this region is very small in comparison to partial waves 5 and 6 . Those reflect a diffraction pattern that is, as expected, located around $\theta^{\prime}=-10^{\circ}$. The global shape of the peak results mainly from the interferences between partial waves 5 and 6 .

As the figures show a good correlation between the models, we can conclude that in the case studied here, our simple FOM is an acceptable tool for further investigation of diffraction at infinity by such structures. Using the software MATLAB, the calculation time for one diffraction pattern, with a $2.33 \mathrm{GHz}$ dual-core CPU personal computer with $8 \mathrm{~Gb} \mathrm{RAM}$, is about 24 hours with the Maxwell-based models; it is a few seconds with the FOM.

\section{LIMITS OF THE MODEL}

Obviously there are certain limits to our model. For instance, considering Fig. 1(b), when the distance $L_{3}$ between the left limit of the partial wave 3 and the $z$ axis becomes close to one wavelength, more differences between FOM and a-FMM appear. This happens when the incident light is close to normal incidence and/or when $H$ is itself on the order of one wavelength or less, and then our approximation of ignoring the effect of the $n_{1}-n_{2}$ interface on the plane waves of the angular decomposition as if their light was propagating only in a homogeneous $n_{2}$ medium $-2 H<z<0$ as shown in Fig. 3,

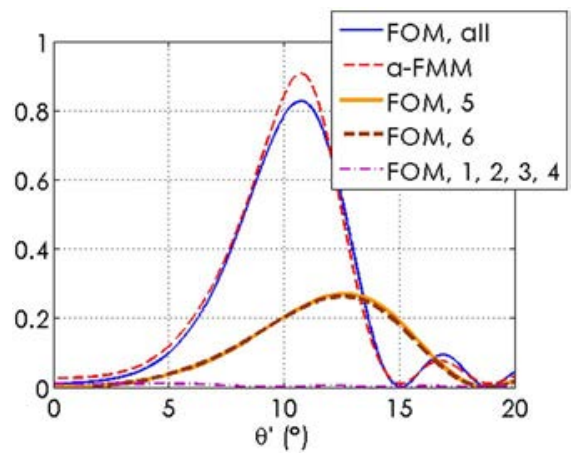

Fig. 7. (Color online) TE mode $\left(n_{1}=1.5, n_{2}=1.4, \quad n_{3}=1\right.$, $H=20 \mu \mathrm{m}, \theta=10^{\circ}$ ). Peak associated to partial waves 5 and 6 , calculated using our FOM, considering case 5 only (thick orange curve), case 6 only (brown, thick, dashed curve), cases 1 to 4 (magenta dashed-dotted curve), and considering cases 1, 2, 3, 4, 5, and 6 (blue solid curve). 
is not valid any more. For instance, we found that the results of the FOM fail if we consider $\theta=1^{\circ}$ with the set of parameters of Fig. 5 , in which case the distance $L_{3}$ is equal to $465 \mathrm{~nm}$, which is lightly smaller than the wavelength. This result meets Swanson's statement [4] that its extended scalar theory applied to multilevel gratings is not acceptable if the period of these gratings is not large in comparison to the wavelength.

A second limit lies in the fact that we consider only one reflection on the back surface of the slab, which is obviously not the case in the a-FMM model. This limit can explain the small difference between the models observed around the peak associated to partial waves $5^{\prime}$ and $6^{\prime}$ in Figs. $\underline{5}-\underline{7}$. In the case of Fig. $\underline{4}, n_{0}=n_{1}$ and $n_{2}=n_{3}$, and therefore no multiple reflection occurs. Then neither the first nor the second limit mentioned applies to that case, and we can see that the curves match perfectly.

\section{CONCLUSION}

In this paper, we have described a simple intuitive $1 \mathrm{D}$ model based on Fourier and elementary geometric optics, to calculate the reflected far-field diffraction pattern of a 1D discontinuity in a thin slab illuminated by an infinite plane wave. This model presents the advantage of being simple to implement and time saving. The results obtained were compared to those from "rigorous" electromagnetic algorithms based on the Fourier modal method and show a good correlation in a range of parameters that we have qualified by examining the limits of validity of our model.

Compared to previous works on advanced heuristic diffraction models since Swanson [4]], this work has introduced the use of the plane wave expansion model of Fourier optics, which is particularly suited to nonperiodic structures. In addition, we have shown that the heuristic decomposition of light diffraction provides an estimate of how much light is diffracted by the various possible sequences of successive reflection and refraction: that has no equivalent in the electromagnetic model. We feel that the panoply of heuristic diffraction techniques can now be used for many other simple structures of interest-it being understood that complex structures are intractable through heuristic approaches and that they cannot address cases where length scales too close to or smaller than the wavelength come into play.

\section{APPENDIX A}

The study of partial waves 1 and 2 is straightforward, as in both cases there is only one reflection, respectively, at $n_{0}-$ $n_{1}$ and $n_{0}-n_{2}$. Let us first consider case 1 . We start from

$$
u_{1}\left(x, 0_{+}\right)=\operatorname{He}(-x) e^{i \frac{2 \pi}{\lambda} n_{0} x \sin \theta} t_{01}(\theta),
$$

where $t_{01}(\theta)$ is the Fresnel transmission coefficient at $n_{0}-n_{1}$ under $\theta$ incidence, and we note $\tilde{u}_{1,0}(\mu)$ the Fourier transform of $u_{1}\left(x, 0_{+}\right)$. Then

$$
u_{1, \infty}(\alpha) \propto \sqrt{\frac{1-\alpha^{2}}{\lambda}} \tilde{u}_{1,0}\left(\frac{\alpha}{\lambda}\right) .
$$

Considering case 2:

$$
u_{2}\left(x, 0_{+}\right)=\operatorname{He}(x) e^{i \frac{2 \pi}{\lambda} n_{0} x \sin \theta} t_{02}(\theta),
$$

$$
u_{2, \infty}(\alpha) \propto \sqrt{\frac{1-\alpha^{2}}{\lambda}} \tilde{u}_{2,0}\left(\frac{\alpha}{\lambda}\right)
$$

In case 4 , the pupil is located at $n_{0}-n_{1}$. When the light meets that pupil, it has already performed a double crossing of the $n_{1}$ medium. Then we start from

$$
u_{4}\left(x, 0_{+}\right)=\operatorname{He}(-x) e^{i \frac{2 \pi}{\lambda} n_{0} x \sin \theta} t_{01}(\theta) r_{13}(\theta) t_{10}(\theta) e^{i \frac{2 \pi}{\lambda} \times 2 n_{1} H \cos \theta_{n 01}},
$$

where $t_{01}(\theta)$ and $t_{10}(\theta)$ are, respectively, the Fresnel transmission coefficients at $n_{0}-n_{1}$ and $n_{1}-n_{0}$ and $r_{13}(\theta)$ is the Fresnel reflection coefficient at $n_{1}-n_{3}$ under initial $\theta$ incidence (the angles used for the Fresnel coefficient calculation are $\theta_{n 01}$ and $\left.\theta_{n 013}\right)$, and we note $\tilde{u}_{4,0}(\mu)$ the Fourier transform of $u_{4}\left(x, 0_{+}\right)$. Then

$$
u_{4, \infty}(\alpha) \propto \sqrt{\frac{1-\alpha^{2}}{\lambda}} \tilde{u}_{4,0}\left(\frac{\alpha}{\lambda}\right) .
$$

The study of partial waves $5,5^{\prime}, 6$, and $6^{\prime}$ assumes the pupil to be the $n_{1}-n_{2}$ interface. The unfolded schemes associated to these cases are represented in Figs. $\underline{8}-\underline{11}$.

In case 5,

$$
u_{5}\left(0_{-}, z\right)=\Pi_{H}\left(z+\frac{3 H}{2}\right) e^{i \frac{2 \pi}{\lambda} n_{1}(2 H+z) \cos \theta_{n 01}} t_{01}(\theta) r_{12}(\theta),
$$

where $\Pi_{H}(x)$ is the $1 \mathrm{D}$ rectangular function of width $H$ and $r_{12}(\theta)$ is the Fresnel reflection coefficient at $n_{1}-n_{2}$ under initial $\theta$ incidence. The Fourier transform of $u_{5}\left(0_{-}, z\right)$ at spatial frequency $\mu$ is given by

$$
\begin{aligned}
\tilde{u}_{5,0}(\mu)= & t_{01}(\theta) r_{12}(\theta) e^{i \frac{2 \pi}{\lambda} 2 H n_{1} \cos \theta_{n 01}} \\
& \times H \operatorname{sinc}\left[H\left(\mu-\frac{n_{1} \cos \theta_{n 01}}{\lambda}\right)\right] e^{i 3 \pi H\left(\mu-\frac{n_{1} \cos \theta_{n 01}}{\lambda}\right)} .
\end{aligned}
$$

Using the angular spectrum decomposition, we only consider the propagation of plane waves in the $(x<0, z>0)$ direction, and then $\alpha_{n}^{\prime}=n_{1} \cos \theta_{n}$ has a positive value. Let $u_{5, r}$ be the complex amplitude repartition in the $n_{1}$ medium:

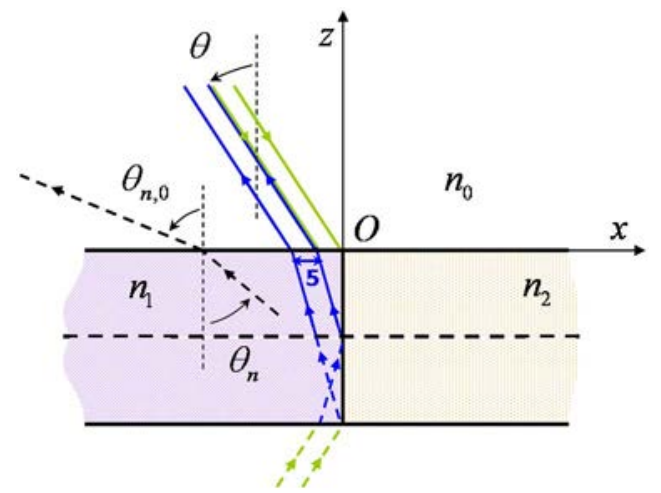

Fig. 8. (Color online) Unfolded scheme associated to the study of partial wave 5 . 


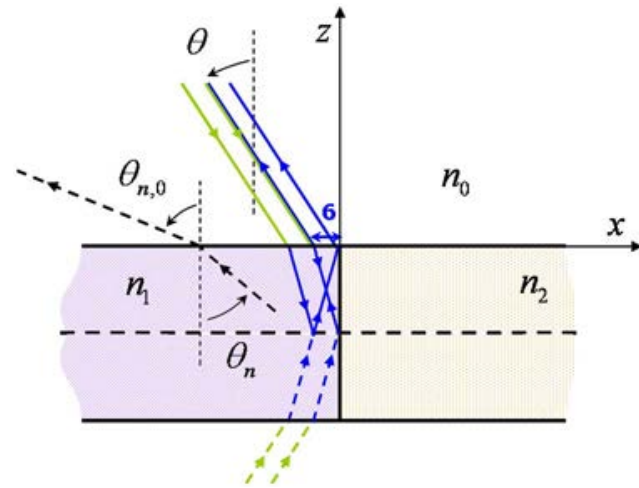

Fig. 9. (Color online) Unfolded scheme associated to the study of partial wave 6 .

$$
u_{5, r}(x, \boldsymbol{z})=\int \tilde{u}_{5,0}\left(\frac{\alpha_{n}^{\prime}}{\lambda}\right) e^{-i \frac{2 \pi}{\lambda} x \sqrt{n_{1}^{2}-\alpha_{n}^{\prime 2}}} e^{i \frac{2 \pi}{\lambda} z \alpha_{n}^{\prime}} \frac{\mathrm{d} \alpha_{n}^{\prime}}{\lambda}
$$

The term below the integral is multiplied by $r_{13}\left(\alpha_{n}^{\prime}\right)$ after reflection at $n_{1}-n_{3}$, where

$$
r_{13, \mathrm{TE}}\left(\alpha_{n}^{\prime}\right)=\frac{n_{1} \cos \theta_{n}-n_{3} \cos \theta_{n, 3}}{n_{1} \cos \theta_{n}+n_{3} \cos \theta_{n, 3}}=\frac{\alpha_{n}^{\prime}-\sqrt{n_{3}^{2}-n_{1}^{2}+\alpha_{n}^{\prime 2}}}{\alpha_{n}^{\prime}+\sqrt{n_{3}^{2}-n_{1}^{2}+\alpha_{n}^{\prime 2}}} .
$$

And then, after refraction at $n_{1}-n_{0}$

$$
\begin{aligned}
u_{5, R T}(x, z)= & \int t_{10}\left(\alpha_{n}^{\prime}\right) r_{13}\left(\alpha_{n}^{\prime}\right) \tilde{u}_{5,0}\left(\frac{\alpha_{n}^{\prime}}{\lambda}\right) \\
& \times e^{i \frac{2 \pi}{\lambda} z \sqrt{n_{0}^{2}-\left(n_{1}^{2}-\alpha_{n}^{22}\right)}} e^{-i \frac{2 \pi}{\lambda} x \sqrt{n_{1}^{2}-\alpha_{n}^{\prime 2}}} \frac{\mathrm{d} \alpha_{n}^{\prime}}{\lambda} .
\end{aligned}
$$

Where here

$$
t_{10, \mathrm{TE}}\left(\alpha_{n}^{\prime}\right)=\frac{2 n_{1} \cos \theta_{n}}{n_{1} \cos \theta_{n}+n_{0} \cos \theta_{n, 0}}=\frac{2 \alpha_{n}^{\prime}}{\alpha_{n}^{\prime}+\sqrt{n_{0}^{2}-n_{1}^{2}+\alpha_{n}^{\prime 2}}} .
$$

In Eq. ( $\underline{\mathrm{A} 11}$ ), the range of values of $\alpha_{n}^{\prime}$ is $\sqrt{n_{1}^{2}-n_{0}^{2}} \leq \alpha_{n}^{\prime} \leq n_{1}$, as the wave vector's norm is equal to $2 \pi n_{0} / \lambda$. Then $t_{10, \mathrm{TE}}\left(\alpha_{n}^{\prime}\right)$ is a real number, whatever the value of $\alpha_{n}^{\prime}$ in this range. As we

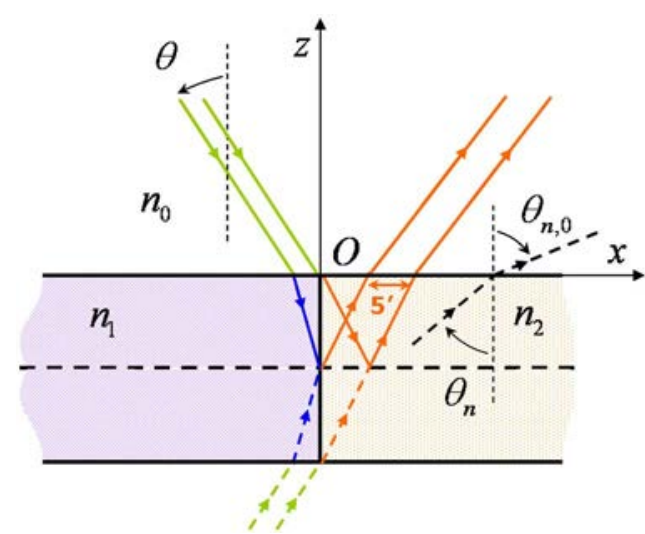

Fig. 10. (Color online) Unfolded scheme associated to the study of partial wave $5^{\prime}$.

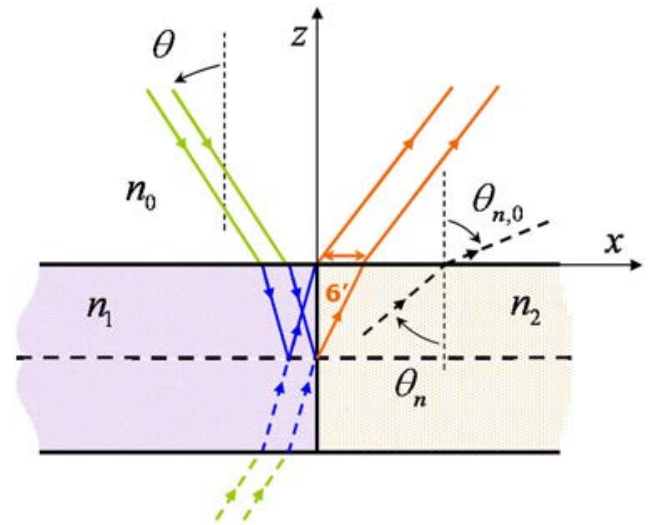

Fig. 11. (Color online) Unfolded scheme associated to the study of partial wave $6^{\prime}$.

already stated it in the study of partial wave 3 , the total internal reflection of the most tilted waves (which correspond to the smallest values of $\alpha_{n}^{\prime}$ ) is then taken into account by this restriction of the range of $\alpha_{n}^{\prime}$ from $0 \leq \alpha_{n}^{\prime} \leq n_{1}$ to $\sqrt{n_{1}^{2}-n_{0}^{2}} \leq \alpha_{n}^{\prime} \leq n_{1}$.

We apply the variable change $v=-\sqrt{n_{1}^{2}-\alpha_{n}^{\prime 2}}$ and then obtain for $u_{5, R T}\left(x, 0_{+}\right)$an analytical expression, here again revealing an inverse Fourier transform. Thus, we can write the repartition at infinity $u_{5, \infty}(\alpha)$ :

$$
\begin{aligned}
u_{5, \infty}(\alpha) \propto & -\sqrt{\frac{1-\alpha^{2}}{\lambda}} t_{10}\left(\sqrt{n_{1}^{2}-\alpha^{2}}\right) r_{13}\left(\sqrt{n_{1}^{2}-\alpha^{2}}\right) \\
& \times \tilde{u}_{5,0}\left(\frac{\sqrt{n_{1}^{2}-\alpha^{2}}}{\lambda}\right) \frac{\alpha}{\sqrt{n_{1}^{2}-\alpha^{2}}}
\end{aligned}
$$

Using the same reasoning for partial wave 6 , we start from

$$
\begin{aligned}
u_{6}\left(0_{-}, z\right)= & \Pi_{H}\left(z+\frac{H}{2}\right) e^{i \frac{2 \pi}{\lambda} n_{1} 2 H \cos \theta_{n 01}} \\
& \times e^{i \frac{2 \pi}{\lambda} n_{1} z \cos \theta_{n 01}} t_{01}(\theta) r_{13}(\theta) r_{12}(\theta)
\end{aligned}
$$

to get the field at infinity:

$$
u_{6, \infty}(\alpha) \propto-\sqrt{\frac{1-\alpha^{2}}{\lambda}} t_{10}\left(\sqrt{n_{1}^{2}-\alpha^{2}}\right) \tilde{u}_{6,0}\left(\frac{\sqrt{n_{1}^{2}-\alpha^{2}}}{\lambda}\right) \frac{\alpha}{\sqrt{n_{1}^{2}-\alpha^{2}}} .
$$

Concerning cases $5^{\prime}$ and $6^{\prime}$, we use the same development with plane waves in the $(x>0, z>0)$ direction:

$$
u_{5^{\prime}}\left(0_{+}, z\right)=\Pi_{H}\left(z+\frac{3 H}{2}\right) e^{i \frac{2 \pi}{\lambda} n_{1}(2 H+z) \cos \theta_{n 01}} t_{01}(\theta) r_{12}(\theta) .
$$

And then 


$$
\begin{aligned}
u_{5^{\prime}, \infty}(\alpha) \propto & -\sqrt{\frac{1-\alpha^{2}}{\lambda}} t_{20}\left(\sqrt{n_{2}^{2}-\alpha^{2}}\right) r_{23}\left(\sqrt{n_{2}^{2}-\alpha^{2}}\right) \\
& \times \tilde{u}_{5^{\prime}, 0}\left(\frac{\sqrt{n_{2}^{2}-\alpha^{2}}}{\lambda}\right) \frac{\alpha}{\sqrt{n_{2}^{2}-\alpha^{2}}}
\end{aligned}
$$

with

$$
\begin{gathered}
t_{20, \mathrm{TE}}\left(\alpha_{n}^{\prime}\right)=\frac{2 \alpha_{n}^{\prime}}{\alpha_{n}^{\prime}+\sqrt{n_{0}^{2}-n_{2}^{2}+\alpha_{n}^{\prime 2}}}, \\
r_{23, \mathrm{TE}}\left(\alpha_{n}^{\prime}\right)=\frac{\alpha_{n}^{\prime}-\sqrt{n_{3}^{2}-n_{2}^{2}+\alpha_{n}^{\prime 2}}}{\alpha_{n}^{\prime}+\sqrt{n_{3}^{2}-n_{2}^{2}+\alpha_{n}^{\prime 2}}} .
\end{gathered}
$$

Finally, for case $6^{\prime}$ :

$$
\begin{aligned}
u_{6^{\prime}}\left(0_{+}, z\right)= & \Pi_{H}\left(z+\frac{H}{2}\right) e^{i \frac{2 \pi}{\lambda} n_{1} 2 H \cos \theta_{n 01}} \\
& \times e^{i \frac{2 \pi}{\lambda} n_{1} z \cos \theta_{n 01}} t_{01}(\theta) r_{13}(\theta) t_{12}(\theta) .
\end{aligned}
$$

And at infinity:

$u_{6^{\prime}, \infty}(\alpha) \propto-\sqrt{\frac{1-\alpha^{2}}{\lambda}} t_{20}\left(\sqrt{n_{2}^{2}-\alpha^{2}}\right) \tilde{u}_{6^{\prime}, 0}\left(\frac{\sqrt{n_{2}^{2}-\alpha^{2}}}{\lambda}\right) \frac{\alpha}{\sqrt{n_{2}^{2}-\alpha^{2}}}$.

\section{REFERENCES}

1. D. M. Cottrell, J. A. Davis, T. R. Hedman, and R. A. Lilly, "Multiple imaging phase-encoded optical elements written as programmable spatial light modulators," Appl. Opt. 29, 2505-2509 (1990).

2. E. Carcolé, J. Campos, and S. Bosch, "Diffraction theory of Fresnel lenses encoded in low-resolution devices," Appl. Opt. 33, 162-174 (1994).

3. M. Peloux, P. Chavel, F. Goudail, and J. Taboury, "Shape of diffraction orders of centered and decentered pixelated lenses," Appl. Opt. 49, 1054-1064 (2010).

4. G. J. Swanson, "Binary optics technology: theoretical limits on the diffraction efficiency of multilevel diffractive optical elements," MIT Technical Report 914 (MIT Lexington Lincoln Lab, 1991).

5. E. N. Glytsis, "Two-dimensionally-periodic diffractive optical elements: limitations of scalar analysis," J. Opt. Soc. Am. A 19, 702-715 (2002).

6. W. Singer and H. Tiziani, "Born approximation for the nonparaxial scalar treatment of thick phase gratings," Appl. Opt. 37, 1249-1255 (1998).

7. M. Testorf, "Perturbation theory as a unified approach to describe diffractive optical elements," J. Opt. Soc. Am. A 16, 1115-1123 (1999).

8. A. v. Pfeil, F. Wyrowski, A. Drauschke, and H. Aagedal, "Analysis of optical elements with the local plane-interface approximation," Appl. Opt. 39, 3304-3313 (2000).

9. H. Wang, D. Kuang, and Z. Fang, "Diffraction analysis of blazed transmission gratings with a modified extended scalar theory," J. Opt. Soc. Am. A 25, 1253-1259 (2008).

10. G. Moulin, F. Goudail, P. Chavel, and D. Kuang, "Heuristic models for diffraction by some simple micro-objects," J. Opt. Soc. Am. A 26, 767-775 (2009).

11. V. Kettunen, M. Kuittinen, and J. Turunen, "Effects of abrupt surface-profile transitions in nonparaxial diffractive optics," J. Opt. Soc. Am. A 18, 1257-1260 (2001).

12. J. W. Goodman, Introduction to Fourier Optics (McGraw-Hill, 1996), Sec. 3.10, pp. 55-60.

13. M. G. Moharam and T. K. Gaylord, "Rigorous coupled-wave analysis of planar-grating diffraction," J. Opt. Soc. Am 71, 811-818 (1981)

14. J. P. Hugonin and P. Lalanne, "Perfectly matched layers as nonlinear coordinate transforms: a generalized formalization," J. Opt. Soc. Am. A 22, 1844-1849 (2005).

15. G. C. Sherman, J. J. Stamnes, and E. Lalor, "Asymptotic approximations to angular-spectrum representations," J. Math. Phys. 17, 760-776 (1976). 